his proper training; and when he comes to use larger quantities of materials and more expensive apparatus he is more likely to do so with economy and care.

In the early part of the work many simple substitutes for the test tube may be used, and there is an advantage in variety, the chief of many considerations being the ease with which such things are washed: a glass slide, as used for the microscope, or any small piece of glass is suitable-for it may be rapidly cleaned after each drop-experiment-and the drop may be studied with the pocket lens, enabling the student to distinguish between crystalline and amorphous precipitates and to note whether the colour is in the precipitate or the solution; indeed the training of the student in the use of the pocket lens from the very outset is highly desirable. Other materials are porcelain tiles or broken china, readily replaced by the student himself. A third class of material, very convenient and possessing certain advantages in chemical tests, is filter-paper or some form of absorbent paper, or in its absence white paper of any kind. When a reaction takes place in a drop on filter-paper, the separation of the precipitate from the solution becomes even more obvious than when the same reaction takes place on a tile or piece of glass. He may learn something also about surface tension, adsorption, and the difference between crystalloid and colloid, and many elementary physical facts which he would never learn by the test tube method.

There are very few reactions commonly carried out in the chemical laboratory which cannot be carried out just as well or better in the microchemical way; for example, the reduction of a copper solution by an aldehyde. Let the student place several separate drops of Fehling's solution on a glass slide and to each add a drop of some different aldehyde solution; let him warm the slide gradually over a small flame and make comparison of the changes in the several drops. This is an example of an experiment of a simple kind where an attempt is made to obtain constant conditions, varying only one factor. Such instructive little tests as these are surely worth far more than mere colour or precipitation tests in test tubes. Many more such tests could be described, but each teacher will probably prefer to devise experiments of his own, suited to the needs of his class.

It is submitted, therefore, that microchemical methods form not only an excellent introductory training for the student but mean a great saving in time, labour, and money. We have saved in one year at least $\mathrm{x}, \mathrm{oool}$., enabling us to buy more apparatus of a permanent kind for teaching and research.

Government Medical School, EgERTON C. GREy. Cairo, July 1922.

\section{An Atomic Model with Stationary Electrons.}

BoHR's atomic model with its circling electrons appears at first sight quite incompatible with Langmuir's model, in which the electrons are stationary or oscillate about fixed positions of equilibrium. Dr. langmuir himself, however, has pointed out that a static atom possessing many of the properties of the Bohr atom is possible provided a force of repulsion equal to $\mathrm{F}=\mathrm{I} / m r^{3} \cdot(n h / 2 \pi)^{2}$ act between an electron (mass $m$, charge $e$ ) and a nucleus. Here $n$ is an integer and $h$ is Planck's constant. The distance $r$ of the electron from the nucleus in stable equilibrium is the same as the radius of a circular orbit corresponding to a stationary state in Bohr's theory. The total energy of the electron is also the same as that given by Bohr's theory. The frequency of oscillation about Publishing Group 21922. the position of equilibrium is identical with the frequency of revolution of the electron in the Bohr atom. Thus the Rydberg constant and the Balmer series can be deduced without assuming moving electrons.

I wish to direct attention to the fact that a force of exactly the type required in Langmuir's theory is provided by the quantum mechanism recently described by Prof. E. T. Whittaker in the Proceedings of the Royal Society of Edinburgh. The mechanism may be pictured as a magnetic wheel consisting of a number of magnetic poles (total strength $M$ ) revolving in a circle of radius $a$. When this magnetic wheel is rotating about its axis with angular velocity $\omega$, it sets up an electric field such that an electron situated at a point on the axis at a distance $r$, large in comparison with $a$, is acted on by a force $\mathrm{Me} a^{2} \omega / r^{3}$ along the axis. Prof. Whittaker has shown that the angular momentum of the magnetic wheel in its steady state (after the passage of an electron completely through it) is determined by $A \omega=2 e \mathrm{M}$, where $A$ is the moment of inertia of the wheel. We shall assume that, in general, the angular momentum is given by Nicholson's quantum relation, so that

$$
\mathrm{A} \omega=2 e \mathrm{M}=n h / 2 \pi \text {. }
$$

Substituting the values of $\mathrm{M} e$ and $\omega$ thus found in the expression for the force on the electron, we find that the "quantum force" is given by

$$
\frac{a^{2}}{2 \mathrm{~A} r^{3}}\left(\frac{n h}{2 \pi}\right)^{2} \text {. }
$$

This agrees precisely with Langmuir's expression for the force of repulsion, provided we make the single additional assumption that $\mathrm{A}=\frac{1}{2} m a^{2}$.

Thus we see that by means of Prof. Whittaker's quantum mechanism it is possible to construct an atomic model which will yield many of the results of Bohr's theory, without employing moving electrons: I have discussed the question more fully in a paper to be published by the Physical Society of London.

August I 5 .

H. S. Allen.

\section{The Variable Depth of Earthquake Foci.}

Prof. H. H. TuRner has given reasons (Mon. Not. R.A.S., Geophys. Suppt. No. I) for believing that the depths of the foci of earthquakes differ among themselves by quantities up to about $300 \mathrm{~km}$. It mav be pointed out that this is precisely what may be expected from the theory of a cooling earth. The available information concerning the thermal state of the earth indicates that the rocks in the asthenosphere, at depths of $400 \mathrm{~km}$. and more, must be very much weaker than those at the surface; this is amply confirmed by the geodetic evidence collected by Barrell, which also suggests that the rocks at depths comparable with $100 \mathrm{~km}$. are considerably stronger than those at the surface. Accordingly, whatever may be the cause of crustal deformation in the earth, yield will occur in the asthenosphere for smaller stresses than are necessary to produce it in the upper parts of the crust. Thus the earthquakes arising from fractures below $400 \mathrm{~km}$. would be more numerous but much less violent than those occurring at higher levels, and the greatest earthquakes should have their foci at the depth of greatest strength. We should therefore expect that the depths of earthquake foci may range from zero to 200 or $300 \mathrm{~km}$.

DOROTHY WRINCH

HAROLD JEFFrEys. 